\title{
On the Detection Possibility of Extragalactic Objects' Redshift Change
}

\author{
Jaroslav Hynecek ${ }^{1}$ \\ ${ }^{1}$ Isetex, Inc., 905 Pampa Drive, Allen, TX 75013, USA \\ Correspondence: Jaroslav Hynecek, Isetex, Inc., 905 Pampa Drive, Allen, TX 75013, USA. E-mail: \\ jhynecek@netscape.net
}

Received: February 11, 2014 Accepted: February 26, 2015 Online Published: February 28, 2015

doi:10.5539/apr.v7n2p58

URL: http://dx.doi.org/10.5539/apr.v7n2p58

\begin{abstract}
This paper describes the detail calculations of expected redshift change in the galaxies' or other distant objects' light after a certain amount of time between observations has elapsed. The detection of this phenomenon has been proposed since the Hubble's discovery of the galaxies' redshift dependence on their distance from Earth and their significant recession velocities. Various astrophysicists have performed such calculations for several cosmological models of the Universe, but not for the model introduced by the author of this paper. This is now addressed in this publication.
\end{abstract}

Keywords: galaxy redshift change in time, finite size model of the Universe, Big Bang theory, gravitational redshift, Doppler redshift, Hubble constant, deformable dark matter, cosmological gravitational potential, gamma ray bursts, CMBR temperature

\section{Introduction}

Several astrophysicists have proposed the measurement of change in the galaxies' redshift with the elapsed time of observations. The most comprehensive and early evaluation of this phenomenon was published by Sandage $(1962,2010)$ with possibly the earliest version suggested by Tolman $(1930,1934)$. Several recent publications on this subject are by Loeb (1998) and by Lerner, Falomo, and Scarpa (2015). However, all of these publications are using some variations of the classical models of the Universe based either on the main stream Big Bang (BB) model or on the Newtonian flat space model.

In the previous publication Hynecek (2012a) has introduced a new model of the Universe that assumes the Universe being finite in size and filled with a repulsive and deformable Dark (transparent) Matter (DM). The DM is repulsive to visible radiating matter but attractive to itself. In this model the galaxies are treated only as small test bodies floating from the bulk of the Universe to the edge where they explode and generate the well-known immense Gamma Ray Bursts (GRB) detected here on Earth. The GRBs that are reflected back to the bulk of the Universe then contribute to the generation of new matter. This model is in line with the theory proposed by Hoyle, Burbidge \& Narlikar (2000) of a steady state Universe with a constant matter creation. The new matter then condenses to stars and eventually to new galaxies endlessly repeating the cycle of creation and destruction. The galaxies' explosions residue at the edge of the Universe generates also the Cosmic Microwave Background Radiation (CMBR) with its temperature of $2.725^{\circ} \mathrm{K}$. The repulsive DM density is very small but its gravitational effects dominate the visible matter gravitation at large distances. The gravitational field of galaxies is thus compensated and screened by the DM after a certain distance. The galaxies thus for the most part move in this Universe independently of each other.

The one of the significant contributions of this model to the theory of the Universe, in comparison to other models of the Universe in particular the BB model, is in the derivation of the relation between the Hubble constant and the CMBR temperature (Hynecek, 2013). These two parameters are typically considered independent of each other. In this model they have been found dependent and one can be derived from the other. This is only possible in a finite and thermodynamically enclosed model as this one is and not in the open models such as the BB.

The details of the model mathematical background and its excellent agreement with the various observations have been published earlier by Hynecek (2012a, 2014). The important equations needed for the derivation of the redshift time dependence are presented in the next section. 


\section{Mathematical Background}

Since the long range gravitational effects of the visible radiating mater and all of the radiation can be neglected, the space-time metric can be considered static, spherically symmetric, and described by the following differential metric line element (Hynecek, 2011, 2012b):

$$
d s^{2}=g_{t t}(c d t)^{2}-g_{r r} d r^{2}-\rho^{2} g_{t t} d \Omega^{2}
$$

where: $d \Omega^{2}=d \vartheta^{2}+\sin ^{2} \vartheta d \varphi^{2}, g_{t t}=\exp \left(2 \varphi_{v}\right), g_{t t} g_{r r}=1$, and $c$ is the local intergalactic speed of light. The cosmological Newton gravitational potential for the visible matter $\varphi_{v}$ normalized to $c^{2}$ is calculated using the well-known equation:

$$
\varphi_{v}(r)=-\frac{4 \pi \kappa}{c^{2} \rho(r)} \int_{0}^{\rho(r)} m(\rho) \rho(r)^{2} d \rho
$$

where $\kappa$ is the Newton gravitational constant. Due to the deformation of the observed natural radius $r$ by the DM gravity, the physical radius $\rho(r)$ must be used in the formula instead of $r$ and this parameter is found from the differential equation that follows from the metric:

$$
d \rho=\sqrt{g_{r r}} d r=e^{-\varphi_{v}} d r
$$

Since any particular galaxy now represents only a small test body in this Universe, the well-known and many times verified Lagrange formalism can be used to describe the motion of such galaxies. The Lagrangian is therefore as follows:

$$
L=e^{2 \varphi_{v}}\left(\frac{c d t}{d \tau}\right)^{2}-e^{-2 \varphi_{v}}\left(\frac{d r}{d \tau}\right)^{2}-\rho^{2} e^{2 \varphi_{v}}\left(\frac{d \Omega}{d \tau}\right)^{2}
$$

For a purely radial motion the Lagrangian can be simplified and the first integrals of the corresponding Euler-Lagrange equations easily found using the initial condition at the origin where the recession velocity is zero and where: $d \tau=d t$. The results are:

$$
\begin{gathered}
\frac{d t}{d \tau}=e^{-2 \varphi_{v}} \\
\left(\frac{d r}{d \tau}\right)^{2}=c^{2}-c^{2} e^{2 \varphi_{v}}
\end{gathered}
$$

Eliminating the non-observable $d \tau$ from these equations then leads to the formula for the recession velocity:

$$
\frac{d r}{d t}=c e^{2 \varphi_{v}} \sqrt{1-e^{2 \varphi_{v}}}
$$

For the relatively near objects, where the cosmological gravitational potential for the visible matter $\varphi_{v}$ is still small, it holds that: $\rho(r) \sim r$, and $m(\rho)=m_{0}$. This simplifies Equation 7 as follows:

$$
\frac{d r}{d t} \cong c \sqrt{-2 \varphi_{v}}=\sqrt{\frac{8}{3} \pi \kappa m_{0}} r=H_{0} r
$$

From this result it is then clear that the recession velocity is linearly proportional to the natural coordinate distance $r$ of such nearby objects from the origin and that the Hubble constant $H_{0}$ is related to the DM density $m_{0}$ at the origin according to the following Equation:

$$
H_{0}=\sqrt{\frac{8}{3} \pi \kappa m_{0}}
$$

The recession velocity and the Hubble constant are referenced to the DM coordinate system, so the value of the Hubble constant should be corrected and referenced to the Earth's centered coordinate system from where it is actually measured. However, the correction is very small and it will be neglected. Earth and its Milky Way galaxy are located relatively near the center of the Universe in comparison to its immense size.

In order to proceed further in the model description it is necessary to find the relation for the DM density $m(\rho)$ as a function of the physical radius. This is obtained by adapting the well-known approach described, for example, 
by Zel'dovich and Novikov (2011) where the DM pressure gradient is expressed as a function of the physical radial distance:

$$
\frac{d P}{d \rho}=-\frac{4 \pi \kappa m(\rho)}{\rho^{2}} \int_{0}^{\rho} m(\rho) \rho^{2} d \rho
$$

After substituting for the DM pressure from the relation: $c=\sqrt{P / m}$, and defining the normalized mass density function: $m_{n}(\rho)=m(\rho) / m_{0}$, Equation 10 can be rearranged with the help of the Green's function as:

$$
m_{n}(\rho)=\exp \left(-A_{0} \int_{0}^{\rho} m_{n}(\xi)\left(\xi-\xi^{2} / \rho\right) d \xi\right)
$$

where $A_{0}$ is a constant equal to: $A_{0}=4 \pi \kappa m_{0} / c^{2}$. There is no known analytic closed form solution for this equation, so it is necessary to use the numerical iterative approach or find an approximating function. The approximating function approach was selected for the next steps to avoid very long computing times during iterations. The selected function, however, underestimates the true value of the DM mass density at large $\rho$, but the error has only a small overall effect. The first two iterations and the approximating function:

$$
m_{a}(\rho)=\exp \left(-\frac{\rho^{2}}{\rho_{h}^{2}}+\frac{3}{10} \frac{\rho^{4}}{\rho_{h}^{4}}-\frac{4}{35} \frac{\rho^{6}}{\rho_{h}^{6}}+\frac{61}{1260} \frac{\rho^{8}}{\rho_{h}^{8}}-\frac{4507}{231000} \frac{\rho^{10}}{\rho_{h}^{10}}+\ldots\right)
$$

are shown in a graph in Figure 1.

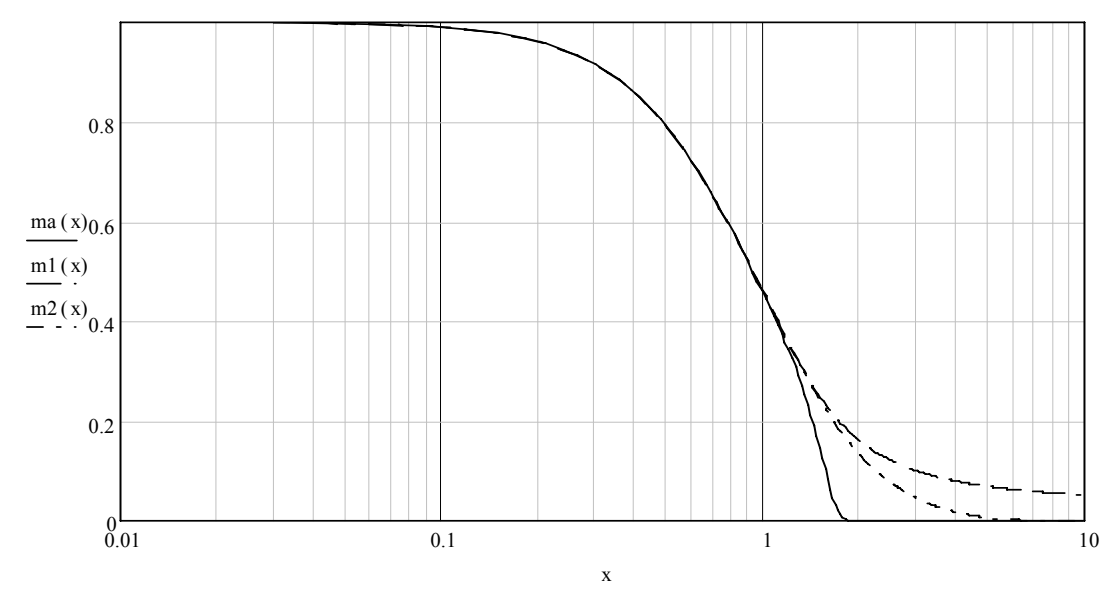

Figure 1. The graphs of the first two iterations (dashed and dot-dashed lines) and the approximating function describing the dark matter mass density as a function of the physical radius where: $x=\rho / \rho_{h}$. The introduced parameter defined as: $\rho_{h}=2 c / H_{0}$, is called the Hubble distance or the Hubble physical radius

Another advantage of using the approximating function is that the DM concentration tail extending past the maximum radial distance can be easily cut off by suitably truncating the power series expansion in the exponent. This feature is advantageous if it is considered that the visible matter debris from explosions of galaxies are accumulating at the edge of the universe and are forming a loosely bound shell there. Of course, it is possible to add more terms than shown in Equation 12; however, this will not be pursued any further in this paper, since the accuracy of the approximation was found reasonable.

Once the mass density function is known it is easy to find the normalized gravitational potential for the visible matter using the formula in Equation 2, and for the dark matter using the Green's function formula derived also from the Gauss law as follows:

$$
\varphi_{d}(\rho)=A_{0} \int_{0}^{\rho} \frac{d \xi}{\xi^{2}} \int_{0}^{\xi} m_{a}(\zeta) \zeta^{2} d \zeta-3.303=A_{0} \int_{0}^{\rho} m_{a}(\xi)\left(\xi-\xi^{2} / \rho\right) d \xi-3.303
$$

Both potentials are plotted in the graphs shown in Figure 2.

In the next step of the model description it is necessary to find the formula for the $Z$ shift, since this is the parameter that is directly measured by astronomers. The $Z$ shift typically consists of the three components: the star gravity 
induced redshift, the cosmological potential induced redshift, and the Doppler redshift resulting from the recession velocity. The star gravity induced redshift does not have to be considered here, since after the star or the galaxy explosions have occurred when the Supernova explosions or the GRB data are compiled, most of the principal source of the gravitational field has been converted to radiation and radiated away and only the remnants or the afterglow produce the light that is observed. The cosmological potential induced redshift does not have to be considered either, since in this model the galaxies are in a radial free fall and this compensates for the shift.

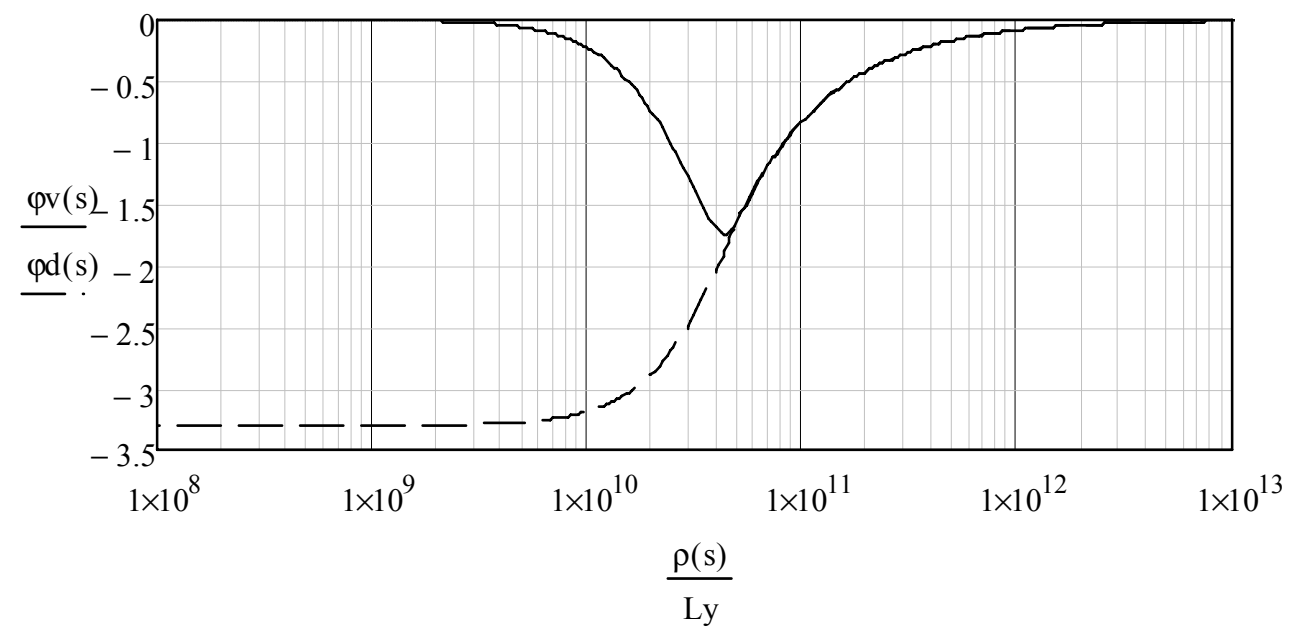

Figure 2. The graphs of dependencies of normalized gravitational potentials for the visible matter (solid line) and for the dark matter as functions of the physical radius. The integration constants were adjusted such that the potentials at infinity are zero

The only remaining redshift component is thus the Doppler redshift resulting from the radial recession velocity $v_{r}$. The Doppler redshift observed on Earth, therefore, is:

$$
Z(\rho)=\frac{\sqrt{1-v_{r}^{2} / c_{r}^{2}}}{1-v_{r} / c_{r}}-1=\frac{\sqrt{g_{t t}}}{1-\sqrt{1-g_{t t}}}-1=\frac{\exp \left(\varphi_{v}\right)}{1-\sqrt{1-\exp \left(2 \varphi_{v}\right)}}-1
$$

where $c_{r}$ indicates the light speed at the galaxy location in reference to Earth. The graph of the $Z$ dependency on the natural coordinate radius $r$ is shown in Figure 3.

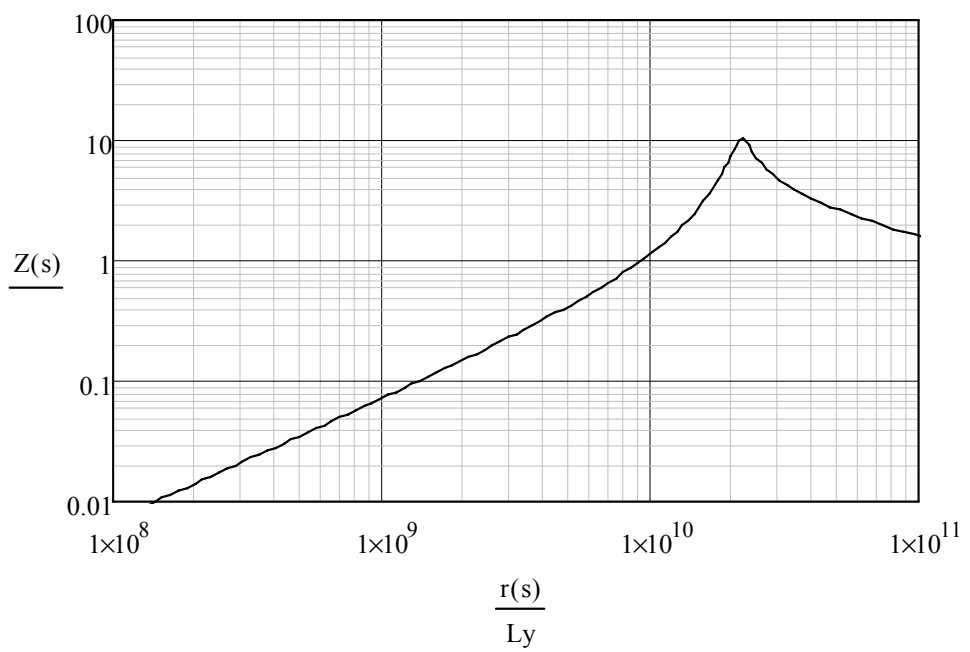

Figure 3. The graph of the dependency of redshift on the natural radial distance $r$. The maximum redshift that can be observed is: $Z_{\mathrm{mx}}=10.35$. The visible matter does not exist at the distances larger than: $r_{m x}=22.11 \cdot 10^{9} L y$, since it disintegrates a the Universe's edge 
The radial distance $r$, also called in this paper the natural radial distance, which is the observable parameter, is calculated according to Equation 3 as follows:

$$
r(\rho)=\int_{0}^{\rho} \exp \left(\varphi_{v}\right) d \rho
$$

To complete the model description the graphs of the galaxies' recession velocity and the speed of light as functions of the natural radial distance $r$ are shown in Figure 4 .

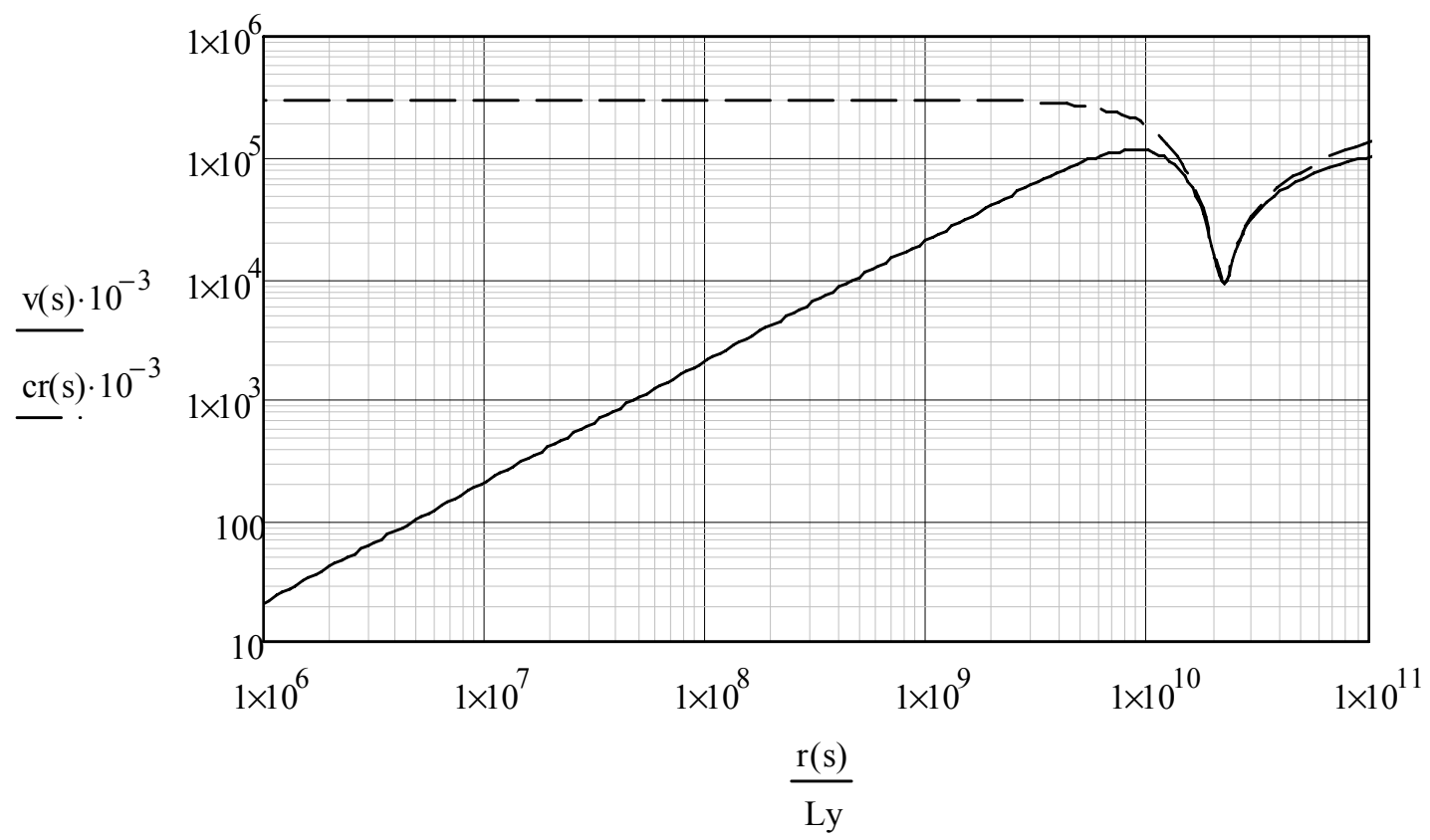

Figure 4. The graphs of the speed of light (dashed line) and the galaxies' recession velocity in $\mathrm{km} / \mathrm{sec}$ as functions of the natural radial distance $r$ from the center of the Universe in light-years

\section{Derivation of the redshift rate change}

The redshift $Z$ dependency on time can be found by first differentiating Equation 14 with respect to distance and then by differentiating the distance with respect to time. The result of differentiating Equation 14 with respect to distance is as follows:

$$
\frac{d Z}{d \rho}=-\frac{d \varphi_{v}}{d \rho} \frac{\exp \left(\varphi_{v}\right)}{\left(1-\sqrt{1-\exp \left(2 \varphi_{v}\right)}\right) \sqrt{1-\exp \left(2 \varphi_{v}\right)}}
$$

The differential of the physical distance with respect to natural time $t$ is found from Equation 3 and Equation 7 .

$$
\frac{d \rho}{d t}=c \exp \left(\varphi_{v}\right) \sqrt{1-\exp \left(2 \varphi_{v}\right)}
$$

By combining Equations 16 and 17 the result for the rate change of the redshift $Z$ is:

$$
\frac{d Z}{d t}=-c \frac{d \varphi_{v}}{d \rho} \frac{\exp \left(2 \varphi_{v}\right)}{1-\sqrt{1-\exp \left(2 \varphi_{v}\right)}}
$$

To proceed further it is useful to find the expression for the potential of the visible matter in terms of the redshift $Z$. This is obtained again from Equation 14 with the result:

$$
\exp \left(\varphi_{v}\right)=\frac{2(Z+1)}{1+(Z+1)^{2}}
$$

Using this formula in Equation 18 results in a suitable expression for the numerical evaluation as follows: 


$$
\frac{d Z_{n}}{d t}=-c \frac{d \varphi_{v}}{d \rho} \frac{2(Z+1)^{2}}{1+(Z+1)^{2}}
$$

It is also possible to find an approximation for the nearby galaxies where the redshift is still small. For this case it holds that the DM density is still reasonably constant and from Equation 2 therefore follows that:

$$
\begin{gathered}
\varphi_{v} \cong-\frac{H_{0}^{2}}{2 c^{2}} \rho^{2} \\
\frac{d \varphi_{v}}{d \rho} \cong-\frac{H_{0}^{2}}{c^{2}} \rho=-\frac{H_{0}}{c} \sqrt{-2 \varphi_{v}}
\end{gathered}
$$

By combining these formulas together, using also the logarithm of formula in Equation 19 to substitute for the visible matter potential, the small $Z$ approximation for the redshift rate change is:

$$
\frac{d Z_{a}}{d t} \cong H_{0} \frac{2(Z+1)^{2}}{1+(Z+1)^{2}} \sqrt{2 \ln \left[\frac{1}{2}\left(Z+1+\frac{1}{Z+1}\right)\right]}
$$

Both of these Equations; 20 and 23 were graphically evaluated using convenient Mathcad 15 numerical symbolic calculations and the results are plotted in graphs in Figure 5.

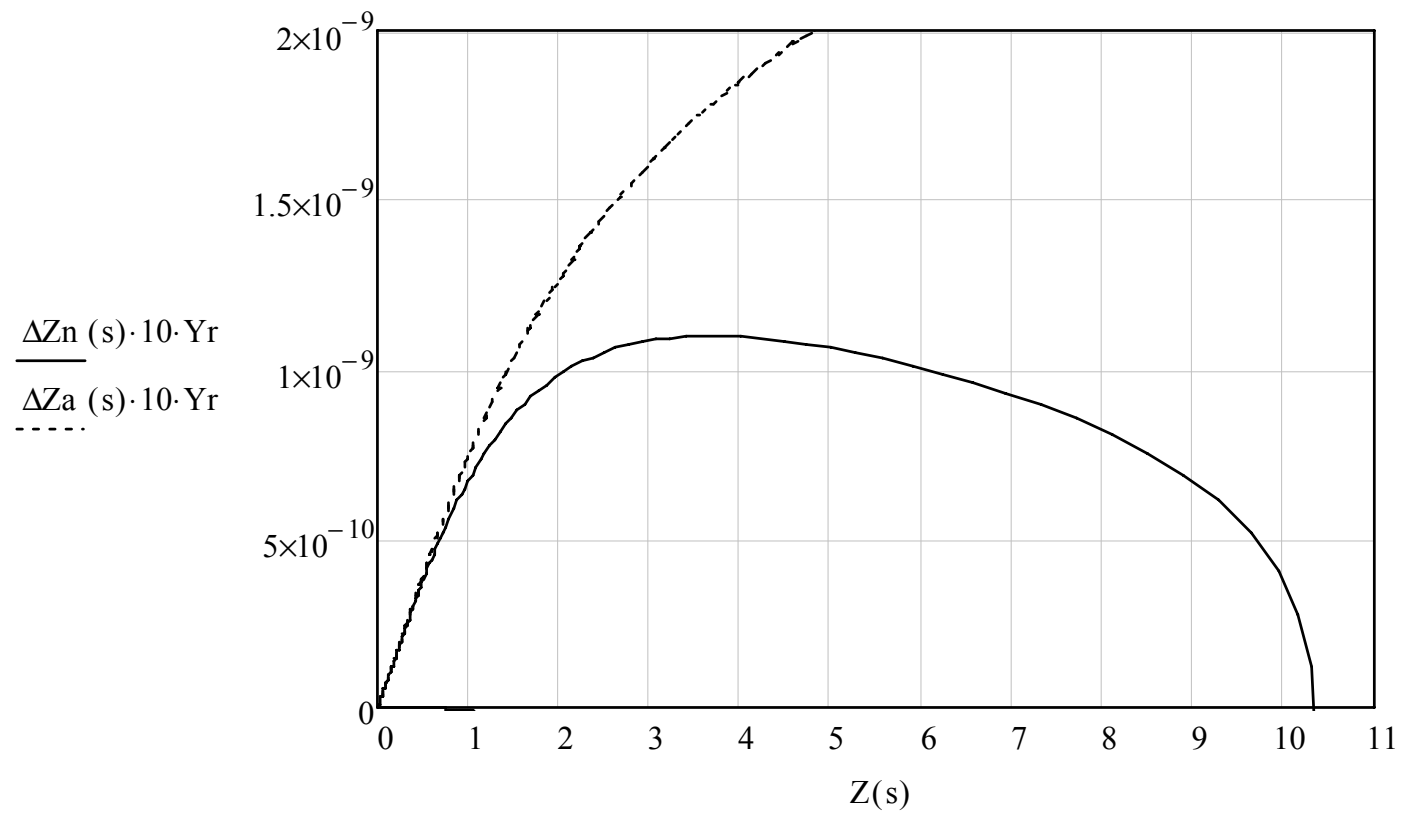

Figure 5. The plots of the dependency of redshift change for the 10 year time period between observations as a function of the galaxy redshift $Z$. The dotted line is the $Z<1$ approximation

The Hubble constant used in all these calculations was: $H_{0}=68.0 \mathrm{~km} / \mathrm{secMpc}$ (Keel, 2007). This value is in an excellent agreement with the value derived by Hynecek (2012a, 2013) from the precisely measured CMBR temperature: $T_{0}=2.725 \pm 0.002{ }^{\circ} \mathrm{K}$. The previously derived formula relating these two parameters is as follows:

$$
T_{H_{0}}=\frac{\exp \left(2 \varphi_{v}\right)}{k_{B}} \sqrt[4]{\left(\frac{3}{8} H_{0}\right)^{2} \frac{h^{3} c^{5}}{\pi \kappa}}=2.727^{\circ} \mathrm{K}
$$

where $k_{B}$ is the Boltzmann constant, $h$ is the Planck constant, and the visible matter cosmological gravitational potential: $\varphi_{v}=-1.7436$ is calculated, using Equations 2 and 12, at the Universe's edge. 
The time interval between the two consecutive observations was selected to be 10 years. From the graphs it is thus clearly seen that for the small $Z$, less than unity, the approximation of constant DM density is reasonable, but fails for the larger values. The interesting result is that there is an optimum redshift rate change for $3<Z<4$ where the redshift rate is the largest. The observations should therefore focus on such cosmological objects. The galaxy deceleration together with the slower speed of light at large distances cause the redshift change rate for large $Z$ objects to be lower and eventually drop to zero at the maximum $Z$ shift: $Z{ }_{\mathrm{mx}}=10.35$. This finding may seem somewhat counterintuitive, since one would expect the larger rate for the larger redshifts. This result is an unavoidable consequence of the finite size model of the Universe that is curved by the DM gravity. Such an interesting finding will most likely not be the same for the BB model or any other similar Universe model derived from the BB theory and, therefore, could be used to confirm the veracity of a particular model.

For a better clarity another plot of the redshift rate change as a function of the natural radial distance $r$ is shown in Figure 6. From this graph it is clear that the redshift rate drops to zero at the edge of the Universe even though the redshift itself is the largest there.

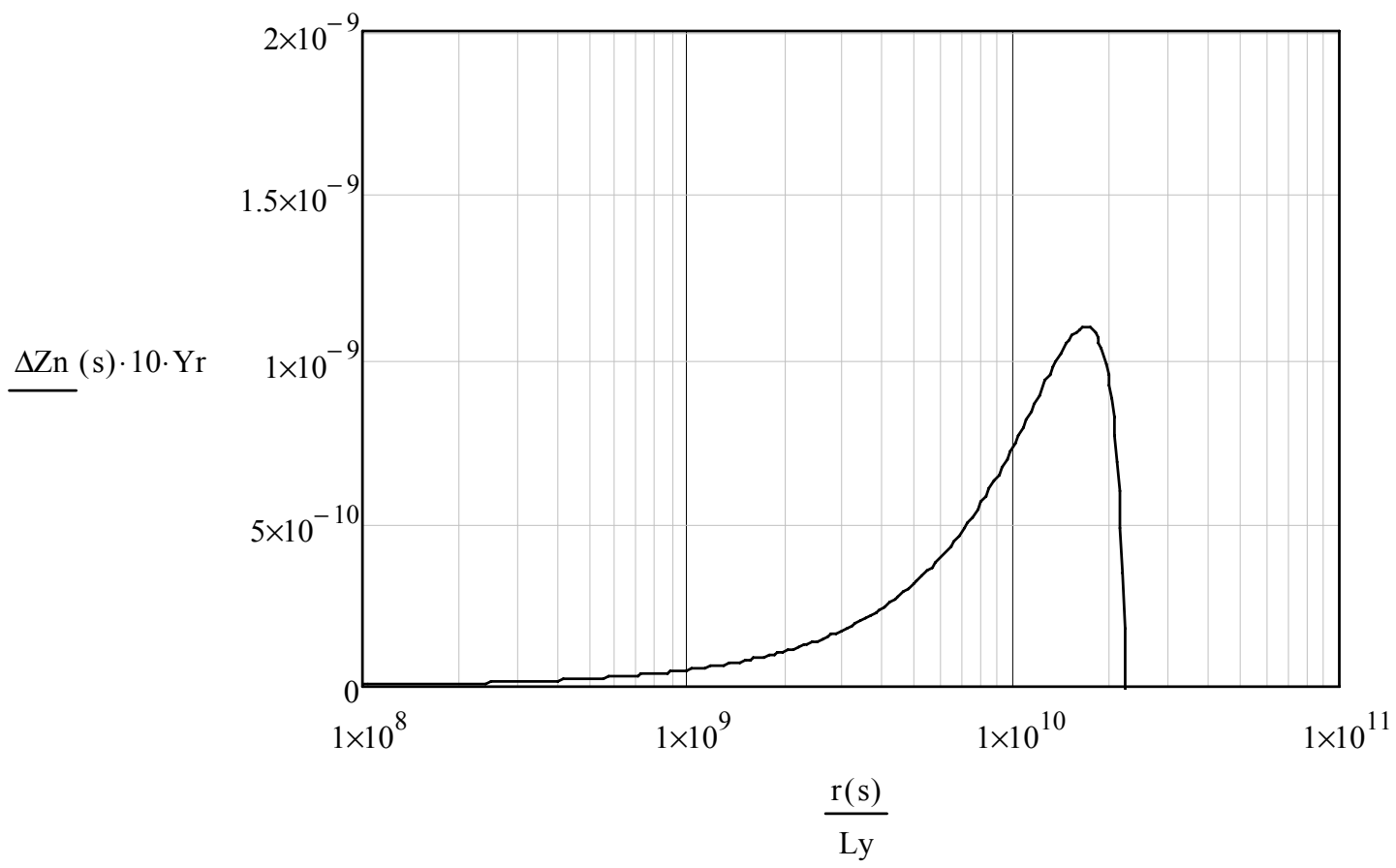

Figure 6. The plot of the dependency of redshift change for the 10 year time period between observations as a function of the natural radial distance $r$ in light-years

\section{Discussion}

It is clear from the graph in Figure 5 that the maximum of the redshift rate that can be observed is not much larger than $10^{-9} / 10 \cdot$ Years. This is an extremely small value that may be difficult to detect. However, with the unprecedented progress in the observational astronomy and in the atomic clock long term stability, the detection might perhaps be possible. It is, of course, necessary to also subtract the rate changes due to the various peculiar motions, such as of Earth in the Sun's orbit, the Sun's motion in our galaxy, and our galaxy motion in the Universe, but this should not present an unsurmountable problem. The reference for subtraction can either be the local galaxy cluster background or the CMBR. Another problem could be the galaxies' intrinsic redshift change. But this effect could also be subtracted by observing the nearby similar galaxies or selected objects. An advantage of the described measurement analysis is that it involves only one variable, the redshift, which can be relatively precisely detected in the light spectrum of distant objects of the Universe.

The successful detection of the redshift rate maximum and the confirmation of the redshift rate dependency on the $Z$ shift as is shown in Figure 5 would represent yet another confirmation of correctness of the finite size model of the Universe, in addition to the determination of the precise value of Hubble constant from the CBMR temperature, and prove the $\mathrm{BB}$ dogma derived from the simplistic extrapolation of observations to be wrong. 


\section{Conclusions}

The article described the detail calculations of the expected redshift change in the galaxies' light after a certain amount of time between the observations has elapsed. This derivation was made for a new model of the Universe. The maximum value of the redshift rate was also derived suggesting the best redshifts at which the observations should be carried out. The possibility of detection of this phenomenon has been proposed since the Hubble's discovery of the galaxies' redshift dependence on their distance from Earth and their significant recession velocities. The detection of this effect would represent a dramatic confirmation of an alternate model of the Universe that is static, finite in space and infinite in time. The Universe without a Big Bang was also recently proposed by Ali and Das (2014).

\section{References}

Tolman, R. C. (1930). On the estimation of distances in a curved universe with a non-static line element. Proceedings of the National Academy of Sciences of the United States of America, 16(7), 511. http://dx.doi.org/10.1073/pnas.16.7.511

Tolman, R. C. (1934). Relativity Thermodynamic and Cosmology. Oxford: Oxford University Press.

Sandage, A. (1962). The Change of Redshift and Apparent Luminosity of Galaxies due to the Deceleration of Selected Expanding Universes. The Astrophysical Journal, 136, 319. http://dx.doi.org/10.1086/147385

Loeb, A. (1998). Direct Measurement of Cosmological Parameters from the Cosmic Deceleration of Extragalactic Objects. The Astrophysical Journal, 499, L111-L114. http://dx.doi.org/10.1086/311375

Hoyle, F. G. Burbidge, F. G., \& Narlikar, J. V. (2000). A Different Approach to Cosmology. Cambridge, U.K.: Cambridge University Press.

Keel, W. C. (2007). The Road to Galaxy Formation (2nd ed., pp. 7-8). Springer.

Sandage, A. (2010). The Tolman surface brightness test for the reality of the expansion. V. Provenance of the test and a new representation of the data for three remote Hubble space telescope galaxy clusters. The Astronomical Journal, 139(2), 728. http://dx.doi.org/10.1088/0004-6256/139/2/728

Zel'dovich, Ya. B., \& Novikov, I. D. (2011). Stars and Relativity. NY: Dover Publications, Inc., Mineola.

Hynecek, J. (2011). Geometry based critique of general relativity theory. Physics Essays, 24(2), 182. Retrieved from http://www.highbeam.com/doc/1G1-258052469.html

Hynecek, J. (2012a). Repulsive dark matter model of the universe. Physics Essays, 25(4), 561. Retrieved from http://www.highbeam.com/doc/1G1-336604030.html

Hynecek, J. (2012b). The Theory of Static Gravitational Field. Applied Physics Research, 4(4), 44-57. http://dx.doi.org/10.5539/apr.v4n4p44

Hynecek, J. (2013). The Repulsive Dark Matter Model of the Universe Relates the Hubble Constant to the Temperature of the CMBR. Applied Physics Research, 5(2), 76-83. http://dx.doi.org/10.5539/apr.v5n2p76

Hynecek, J. (2014). An Interesting Journey of Discovery of Many Errors in Einstein's General Relativity Theory. Retrieved from http://vixra.org/abs/1407.0136

Ali, A. F., \& Das, S. (2014). Cosmology from quantum potential. Retrieved from http://arxiv.org/abs/1404.3093v3

Lerner, E., Falomo, R., \& Scarpa, R. (2015). U V surface brightness of galaxies from the local Universe to Z 5. International Journal of Modern Physics D (to be published). Retrieved from http://arxiv.org/abs/1405.0275

\section{Copyrights}

Copyright for this article is retained by the author(s), with first publication rights granted to the journal.

This is an open-access article distributed under the terms and conditions of the Creative Commons Attribution license (http://creativecommons.org/licenses/by/3.0/). 